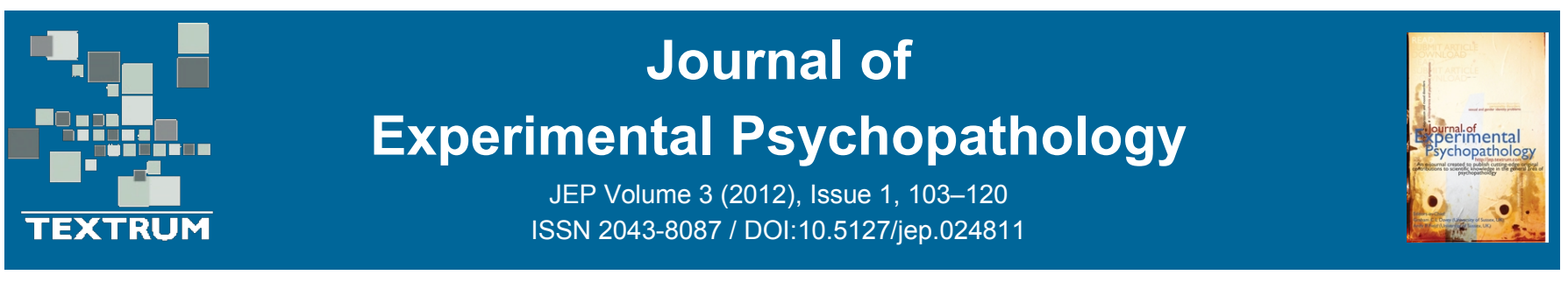

\title{
An Investigation of the Role of Attention in Mindfulness- Based Cognitive Therapy for Recurrently Depressed Patients
}

\author{
Paul A. M. van den Hurk ${ }^{a}$, Joël R. van Aalderen ${ }^{b}$, Fabio Giommi ${ }^{a}$, Rogier A. R. T. \\ Donders $^{c}$, Henk P. Barendregt ${ }^{a}$, and Anne E. M. Speckens ${ }^{b}$ \\ ${ }^{a}$ Faculty of Science, Radboud University, The Netherlands. \\ ${ }^{b}$ Department of Psychiatry, Radboud University, The Netherlands. \\ ${ }^{c}$ Department of Epidemiology, Biostatistics and HTA, Radboud University, The Netherlands.
}

\section{Abstract}

Mindfulness-based cognitive therapy (MBCT) has been shown to be efficacious in reducing relapse rate and depressive symptoms in patients with recurrent depression. To date, little is known about the underlying cognitive mechanisms. We investigated the role of attention with the attention network test in a randomized controlled trial with $34 \mathrm{MBCT}$ patients and 37 waiting-list control patients. In the MBCT group depressive symptoms and ruminative thinking decreased and mindfulness skills increased. However, no differential changes in either specific, basal components of attentional processes (alerting, orienting and executive attention) or more general attentional functioning were observed. These results seem to fit in the pattern, emerging from recent research findings, that suggests that it might be especially the second component of mindfulness as described by Bishop (Bishop et al., 2004) - a shift towards an attitude with more openness and acceptance - that mediates the efficacy of short-term mindfulness interventions.

(c) Copyright 2012 Textrum Ltd. All rights reserved.

Keywords: mindfulness-based cognitive therapy; attention; depression; mindfulness; mediation; acceptance

Correspondence to: Paul A.M. van den Hurk, Institute for Computing and Information Sciences, Radboud University Nijmegen, Heyendaalseweg 1356525 AJ Nijmegen. Email: p.vandenhurk@cs.ru.nl

1. Faculty of Science, Radboud University Nijmegen, Heyendaalseweg 135, 6525 AJ Nijmegen, The Netherlands.

2. Department of Psychiatry, Radboud University Nijmegen Medical Centre, PO Box 9101, 6500 HB Nijmegen, The Netherlands.

2. Department of Epidemiology, Biostatistics and HTA, Radboud University, Nijmegen Medical Centre. PO Box 9101, 6500 HB Nijmegen, The Netherlands.

Received 06-Jan-2011; received in revised form 29-Sep-2011; accepted 07-Oct-2011 


\title{
Table of Contents
}

\author{
Introduction \\ Method \\ Participants \\ Procedure \\ Intervention \\ Measures \\ Hamilton Rating Scale for Depression (HRSD) \\ Rumination on Sadness Scale (RSS) \\ Kentucky Inventory of Mindfulness Skills (KIMS) \\ Attentional Network Test \\ Attention Networks Calculations and Analyses \\ Statistical analyses \\ Depression, rumination and mindfulness skills scores \\ Attention network scores \\ Overall attentional processing \\ Results \\ Depression, rumination and mindfulness skills scores \\ Attention Networks analyses \\ Normalized RT scores \\ Error scores \\ Overall attentional performance \\ Probability of detections and misses \\ Probability of correct and incorrect responses \\ Discussion \\ Concluding remarks \\ Acknowledgements \\ References
}

\section{Introduction}

Over the last decade, mindfulness-based cognitive therapy (MBCT) has been shown to be efficacious in reducing relapse rates and depressive symptoms in patients with recurrent depression (Kuyken et al., 2008; Ma \& Teasdale, 2004; Segal et al., 2010; Teasdale et al., 2000). MBCT is an 8-week group program based on an integration of elements of cognitive-behavioral therapy (Beck, Rush, Shaw, \& Emery, 1979) with the mindfulness-based stress reduction (MBSR) program (Kabat-Zinn, 1990). So far, research has mainly been focused on the effect of MBCT on clinical outcome variables, such as relapse rates and depressive symptoms (Kuyken, et al., 2008; Ma \& Teasdale, 2004; Segal, et al., 2010; Teasdale, et al., 2000). Only few studies have investigated (changes in) cognitive processes that could subserve, mediate these clinical improvements (Anderson, Lau, Segal, \& Bishop, 2007; Chambers, Lo, \& Allen, 2008; Giommi et al., 2006; Heeren, van Broeck, \& Philippot, 2009; Jha, Krompinger, \& Baime, 2007; Kuyken et al., 2010). Therefore, to date, only a limited and preliminary understanding in the underlying mechanism(s) driving the beneficial effect of MBCT has been obtained.

To our knowledge, Heeren et al. (2009) have been among the first (Anderson, et al., 2007; Chambers, et al., 2008; Giommi, et al., 2006; Heeren, et al., 2009; Jha, et al., 2007) to study important changes in cognitive processes during short-term mindfulness trainings such as MBCT. The aim of the study by Heeren et al. (2009) was to examine the role of executive processes, which are necessary when a situation requires more than a routine execution of automatic and overlearned schemata, on the impact of mindfulness on autobiographical memory specificity. Their results suggested that the effects of 
mindfulness training on autobiographical memory specificity are mediated by executive processes and, further, that effects of mindfulness training are enhanced by cognitive flexibility (the ability to switch behavioral response according to the context of the situation).

Besides changes in the cognitive processes mentioned above, changes in attentional processing might also be expected after MBCT, for the training of attention is one of the core practices in the program. The following description of the mindfulness practice during MBCT by Teasdale, Segal, Williams (1995, p.33) illustrates the central role of attention training: "In formal mindfulness practice, the student sits quietly in an erect and dignified posture and attempts, non-strivingly, to maintain attention on a particular focus, commonly his or her own breathing. When the attention wanders from the breath to the thoughts and feelings that inevitably arise, the student 'acknowledges and accepts' the thoughts or feelings, 'lets go' of them, and gently re-directs attention back to the breath. This procedure is repeated many times, whenever the student notices that the attention has wandered. In informal practice, students apply the same general approach as often as possible during the course of their normal day, bringing the attention back to the 'here and now', using a focus on the breath as an 'anchor', whenever they notice that attention has been diverted to streams of thought, worries, reverie, or general lack of awareness". Given this central focus on the training of attention during MBCT, one could expect participation in the program to result in an improved self-regulation of attention, i.e., a more flexible disengaging from irrelevant stimuli and improved general attentional processing of relevant stimuli. Our interest is in whether such improvements in attention have a mediating role in the efficacy of the MBCT program.

In recent years, the effect of mindfulness training on attentional processing has been investigated at the level of specific components of attention (alerting, orienting and executive attention) as well as at a more integrated level of general attentional processing (Anderson, et al., 2007; Chambers, et al., 2008; Chan \& Woollacott, 2007; Jha, et al., 2007; Moore \& Malinowski, 2009; Slagter et al., 2007; Van den Hurk, Giommi, Gielen, Speckens, \& Barendregt, 2010) and a distinction between short-term (like MBCT) and long-term (very intense training for months or years of experience) trainings seems warranted. In studies on the effect of short-term trainings no improvements in specific components of attention have been found (Anderson, et al., 2007; Chambers, et al., 2008; Giommi, et al., 2006). On the contrary, the longterm practice of mindfulness meditation has been associated with improvements in specific, basal components of attention (Chan \& Woollacott, 2007; Slagter, et al., 2007; Van den Hurk, et al., 2010). For example, in the study by van den Hurk et al. (2010), expert, long-term mindfulness meditators showed improvements in orienting and executive attention. Both short-term and long-term trainings have been associated with improvements in attention at a more general, integrated level (Anderson, et al., 2007; Chambers, et al., 2008; Van den Hurk, et al., 2010). For example, in the study by van den Hurk et al. (2010) an improved overall performance on the attention network test (ANT) was found, as reflected by the lower amount of errors made by the meditators for equally fast responses. Importantly, only healthy participants have been tested in all the studies mentioned above and, to our knowledge, just one study has been done that investigated the effect of mindfulness training on attention in clinically depressed and anxious patients (Giommi, et al., 2006). Therefore, to date, it is not clear whether a clinical sample of recurrently depressed patients shows improvements in attention after either short-term or long-term mindfulness trainings.

Since impairments in attentional processing are a hallmark in the psychopathology of depression (Joormann \& Gotlib, 2007; Koster, De Lyssnyder, Derakshan, \& De Raedt, 2010; Merriam, Thase, Haas, Keshavan, \& Sweeney, 1999), there seems room for improvement in attentional processing in recurrently depressed patients. Given this idea of room for improvement in attention and considering the fact that attentional training is a central element in the MBCT program, we hypothesized that MBCT might result in improvements in attention in a sample of recurrently depressed patients, both remitted 
and currently depressed. In more detail, in a recent paper on the underlying cognitive mechanisms of depressive rumination by Koster et al. (2010), the 'impaired disengagement' hypothesis was proposed. According to the authors the core tenet of their model is that prolonged processing of self-referent material is due to impaired attentional disengagement from negative self-referent information. In light of this impaired attentional disengagement underlying depressive rumination and the central role of improving the self-regulation of attention during mindfulness practice, we hypothesized to find specific improvements in orienting and executive attention after MBCT. Both these components of attention are related to the concept of disengaging attention from irrelevant information and directing attention to relevant information. In addition, considering the hypothesized improvements in general attentional processing, we expected improvements in general attentional performance after MBCT. Finally, we were interested in whether such improvements in attention have a mediating role in the efficacy of the MBCT program.

In order to probe both orienting and executive attention as well as more general, overall attentional processing, the ANT was used (Fan, McCandliss, Sommer, Raz, \& Posner, 2002). The ANT probes the functioning of three separate components of attention: i.e. the alerting, orienting and executive attentional networks. Alerting is defined as achieving and maintaining an alert state; orienting is the selection of information from sensory input; and executive control is defined as resolving conflict among responses (Fan, et al., 2002). In addition, the overall performance on the ANT - in terms of speed and accuracy in responding and target detection rate - provides a more general and integrated view of attentional functioning (Van den Hurk, et al., 2010). The current study was embedded in a larger randomized controlled trial comparing MBCT and a treatment as usual (TAU) condition in 205 patients with recurrent depression. In order to probe changes in depressive symptoms, severity of rumination and mindfulness skills, by means of questionnaires, depression, rumination and mindfulness skills scores were obtained.

To summarize, MBCT has been shown to be efficacious in reducing relapse rates and depressive symptoms in patients with recurrent depression (Kuyken, et al., 2008; Ma \& Teasdale, 2004; Z. Segal, et al., 2010; Teasdale, et al., 2000). Yet, very little is known about the (changes in) cognitive processes that subserve, mediate these effects. It is well known that impairments in attention are a hallmark in the psychopathology of depression (Joormann \& Gotlib, 2007; Koster, et al., 2010; Merriam, et al., 1999), suggesting room for improvement in attentional functioning in recurrently depressed patients. Considering the fact that attention training is a core practice in the MBCT program, we predicted to find improvements in attention in a sample of recurrently depressed patients after MBCT. In more detail, improvements in orienting and executive attention were expected, which are components of attention related to disengaging attention from irrelevant information and directing attention to relevant information. Improvements in these attention networks would be shown by decreased orienting and executive attention network scores on the ANT. In addition, in light of the hypothesized improvements in general attentional processing due to the mindfulness practice, we expected to find greater improvements in overall attentional processing for the MBCT group. Such improvements would be shown by enhanced target detection and increased response accuracy after MBCT. We hypothesized that such improvements in attentional functioning have a mediating role in the efficacy of the MBCT program. Finally, in accordance with previous studies (Kenny \& Williams, 2007; Kingston, Dooley, Bates, Lawlor, \& Malone, 2007; Michalak, Heidenreich, Meibert, \& Schulte, 2008), we predicted to find decreased depression and rumination scores and increased mindfulness scores for the MBCT group, which would show the efficacy of the MBCT program. Altogether, this study shows our attempt to investigate the role of attention in the efficacy of $\mathrm{MBCT}$ and hence it seems an adequate response to the 
recent suggestion by Kuyken and colleagues who indicated that future studies should evaluate different dimensions of mindfulness including attentional control in the context of MBCT (Kuyken, et al., 2010).

\section{Method}

\section{Participants}

This study was part of a larger clinical randomized controlled study comparing MBCT (+ TAU) and a TAU condition in patients with recurrent depression (Van Aalderen et al., In submission). Patients were recruited by media announcements or referred by their general practitioner or psychiatrists and psychologists in and around the city of Nijmegen. Alternatively, they were self-referred, informed by local and national advertisements. Patients were then screened by telephone and, if applicable, invited for a research interview including the Mini-International Neuropsychiatric Interview (MINI) (Sheehan et al., 1998; Van Vliet, Leroy, \& Van Megen, 2000), including the section on recurrent depression according to the Structural Clinical Interview for DSM-IV axis I Disorders (SCID-I) (First, Spitzer, Gibbon, \& Williams, 1995; Groenestijn, Akkerhuis, Kupka, Schneider, \& Nolen, 1999). The research interviews were used to confirm in- and exclusion criteria, and were conducted by a psychologist or psychiatrist in training, supervised by an experienced psychiatrist. Patients with three or more previous depressive episodes according to DSM-IV criteria (APA, 2000) were eligible for the study. Patients with a hypomanic or manic episode, psychotic symptoms or severe substance abuse were excluded. A random subset $(n=71)$ of the initial study population $(n=205)$ was asked to participate in the attention network test (ANT; described in detail below) and agreed upon participation in the experiment. With respect to age, gender, baseline level of depression and depression diagnosis the group of participants that agreed upon participation was representative for the whole group of participants that was studied. The MBCT group consisted of 34 patients [mean (SD) age 49.8 (12.2), range 24 - 84 years, 11 male], whereas the control group (TAU condition) consisted of 37 patients [mean (SD) age 49.2 (10.0), range 32 - 71 years, 9 male]. Sixteen patients $(47 \%)$ in the MBCT group and 17 patients $(46 \%)$ in the control group had prior experience with meditation. Twenty-two patients (65\%) in the MBCT group and 25 patients (68\%) in the control group took medication at the start of the treatment. Groups did not differ on age, $t(69)=.208, p=$ .836; gender $\chi^{2}(1)=.564, p=.452$; meditation experience $\chi^{2}(1)=.009, p=.925$; or medication intake at start of treatment $\chi^{2}(1)=.006, p=.936$. Of note, 14 participants in the TAU group and 11 participants in the MBCT group were at baseline diagnosed with a current depression according to DSM-IV axis I Disorders criteria (NS). No significant difference between groups was present regarding the number of participants diagnosed with a current depression, $\chi^{2}(1)=.234, p=.629$.

\section{Procedure}

At first contact, patients were asked to sign an informed consent form. Patients were requested to keep their antidepressant medication stable for the duration of the study and to refrain from any additional psychological treatment. After being assessed for suitability, participants were randomized into either the MBCT group or the TAU condition. Randomization was stratified for Hamilton (HRSD) depression scores of lower than 10 or of 10 or higher (Hamilton, 1960).

All participants were asked to fill out a number of self-report questionnaires before completing the ANT. Assessments took place directly before and after the training/ 3-months control period. Afterwards, MBCT was also offered to patients in the TAU condition. 


\section{Intervention}

MBCT was offered according to the protocol developed by Segal and colleagues (Z. V. Segal, Williams, \& Teasdale, 2002). It consisted of 8 weekly sessions of 2.5 hours each, with an all-day retreat between the 6th and 7th session. The MBCT training was taught by three different trainers (one male, two female). One of the teachers was a consultant psychiatrist and cognitive behavioral therapist with 5 years of meditation experience, 4 years experience guiding MBCT groups who trained at the Insight Meditation Society (IMS; Barre, MA, USA). Another teacher was an occupational therapist with 26 years of meditation experience and 1.5 years experience guiding MBCT groups. The other teacher is a clinical psychologist with 21 years of meditation experience and 3 years experience guiding MBCT groups. The groups consisted of up to 15 participants each.

\section{Measures}

\section{Hamilton Rating Scale for Depression (HRSD)}

The HRSD (Hamilton, 1960) was used to assess depressive symptoms. The HRSD is a 17-item interview-based measure, widely used in clinical care. The Dutch version of the HRSD has shown good internal consistency, Cronbach's $\alpha=0.82$ (Hermans, van Ree, Betgem, \& Westenbrink, 1981).

\section{Rumination on Sadness Scale (RSS)}

The level of rumination was measured with the RSS (Conway, Csank, Holm, \& Blake, 2000). The RSS is a self-report questionnaire measuring ruminative response to depressed mood on 13 items, scored on a 5-point Likert-type of scale. The Dutch translation of the RSS by Raes, Hermans, \& Eelen, (2003) showed good psychometric properties, Cronbach's $\alpha=0.84$ (Raes et al., 2003).

\section{Kentucky Inventory of Mindfulness Skills (KIMS)}

The KIMS (Baer, Smith, \& Allen, 2004) was used to measure levels of mindfulness skills and has been shown to be applicable in clinical populations (Baum et al., 2010). This questionnaire consists of 39 items measuring four domains (observing, act-with-awareness, describing, and accepting-withoutjudgment), consistent with four of the five mindfulness facets as identified by Baer et al. (Baer, Smith, Hopkins, Krietemeyer, \& Toney, 2006). Participants indicated their responses on a Likert-type scale from 1 (almost never) to 5 (almost always). A comparable Dutch version of the KIMS has been shown good psychometric properties, Cronbach's $\alpha=.77-.91$ for the different subscales (Dekeyser, Raes, Leijssen, Leysen, \& Dewulf, 2008).

\section{Attentional Network Test}

Participants were seated in front of a computer screen at a distance of $65 \mathrm{~cm}$. Stimuli were presented and responses were collected with Presentation software (version 10.1 Neurobehavioral Systems, Albany, USA). Participants were instructed to respond as fast and as accurately as possible to a target stimulus that was presented in the center of a horizontal row with five stimuli (fig.1C). The target stimulus was an arrow pointing either to the left or to the right and was flanked by two flanker stimuli on each side. Participants were instructed to press the left mouse button with their left thumb or the right mouse button with their right thumb as fast as possible when the target arrow pointed to the left or right, respectively. The four surrounding flanker stimuli were all arrows pointing in the same or the opposite direction of the target stimulus or were just neutral stripes. The condition in which all five arrows pointed in the same direction was called the congruent target condition. The condition in which the flanker arrows pointed in the direction opposite to the target arrow was named the incongruent target condition. The condition 
when the four flanker stimuli were stripes was called the neutral target condition. The target stimulus and the flanker stimuli were presented at a visual angle of 1.1 above or below a fixation cross presented in the middle of the screen.

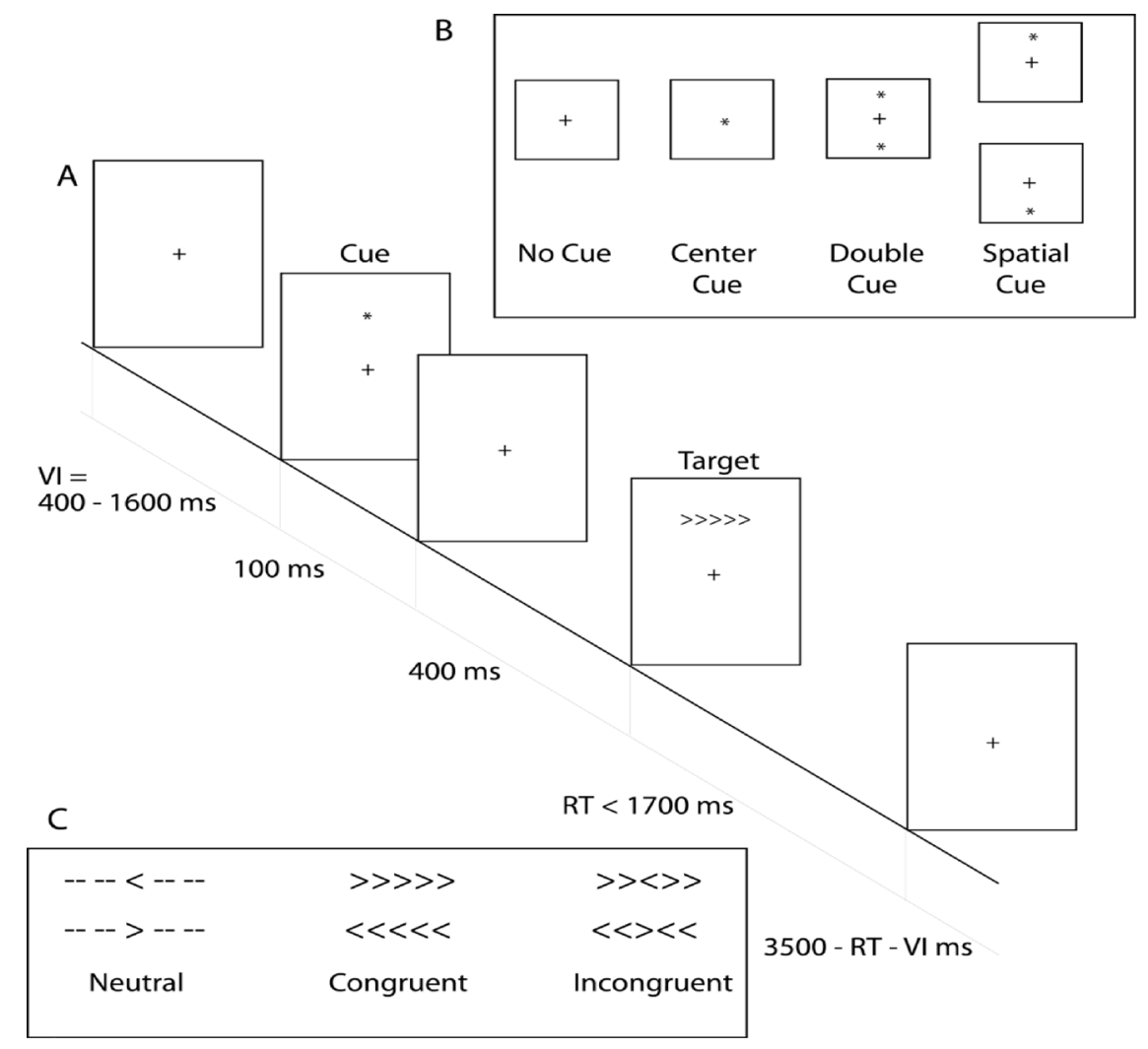

Figure 1: A) During a variable interval (VI $=400-1600 \mathrm{~ms})$ a central fixation cross is presented and the participant is instructed to look at it. Then a cue can be presented for $100 \mathrm{~ms}$. In part B the four cue conditions are shown: No Cue, Central Cue, Double Cue, and Spatial Cue. After presentation of the cue, a central fixation cross is shown and is followed by the target stimulus. In part $C$ the three different target configurations are depicted: the Neutral, Congruent and Incongruent target configurations. The target is depicted until the participant responds with a maximum of $1700 \mathrm{~ms}$. If the reaction time (RT) is shorter than $1700 \mathrm{~ms}$, the stimulus is replaced by the central fixation cross.

The target stimulus could be cued in four different ways (see Figure 1B). In the first cueing condition, an asterisk was presented at the location of the fixation cross (= center cue condition) and the target configuration was presented above or below the center of the screen, with equal probability. In the second cueing condition, two asterisks were presented (= double cue condition); the two asterisks were presented at the fixed locations of 1.1 of visual angle above and below the center of the screen. Since the cue appeared $500 \mathrm{~ms}$ before target onset (see Figure 1A), the cue provided information on the timing of the target stimulus. In the third cueing condition, an asterisk was presented at the future location of the target stimulus above or below the center of the screen (= spatial cue condition). In this case, participants were informed both on the timing and the location of the target configuration. In the fourth cueing condition, no cue was given and, as a consequence, participants had no information about the timing and the location of the upcoming target symbol.

The attention network test consisted of one training block with 24 trials and three test blocks with 96 trials each. After the first and second block, participants took a short break, before starting the next block of the attention network test. A single trial consisted of the following: during a variable interval (VI, see Figure 1A), ranging from $400-1600 \mathrm{~ms}$, a fixation cross was presented in the middle of the screen. Then, depending on the cue condition, a cue could be presented for $100 \mathrm{~ms}$. Thereafter, a central 
fixation cross was presented for $400 \mathrm{~ms}$, followed by the target stimulus, which was presented for 1700 $\mathrm{ms}$, or shorter if a response was given within $1700 \mathrm{~ms}$. Finally, a fixation cross was presented during a variable delay. The length of this delay was determined by subtracting the reaction time (RT) plus 400 $\mathrm{ms}$ from the constant trial duration that was $3500 \mathrm{~ms}$ (fig. 1A). All 12 combinations of cueing (4) and target (3) conditions were presented in random order within each block. Both RT and error scores (ES; percentage of incorrect responses) were measured for each condition.

\section{Attention Networks Calculations and Analyses}

For each participant, all RT of correct responses of a specific condition outside the range of the mean RT $\pm 2.5 \mathrm{SD}$ of that specific condition and RT shorter than $100 \mathrm{~ms}$ were excluded from analysis. Then, the average RT for each of the 12 conditions (4 cue conditions $\times 3$ target conditions) was recalculated. Determination of the network effects was based on these RT data. ES of the 12 conditions were calculated by dividing the number of incorrect responses by the total number of responses for the specific condition and multiplying this number by 100 .

To calculate the alerting network effect mean RT and ES of the double cue condition were subtracted from the mean RT and ES of the no cue condition. In such a way, the potentially beneficial effect of an alerting cue on RT and ES was probed. The orienting network effect was calculated by subtraction of the mean RT and ES of the responses in the spatial cue condition from the mean RT and ES of the responses in the center cue condition. This allowed us to probe the beneficial effect of spatial information, in addition to timing information. The executive network effect was calculated by subtracting the mean RT and ES in the congruent target condition from the mean RT and ES in the incongruent target condition. In this way, the advantage of congruence over incongruence in the target condition was determined. Mean RT and ES of the three target conditions were averaged to calculate the orienting and alerting network effects, whereas mean RT and ES of the 4 cueing conditions were averaged to calculate the executive network effect. Normalized network effects were determined by dividing the raw network effect by the mean of the two conditions involved in the calculation of the network effect. (Raw effect: $R=R T_{A}-R T_{B}$; Normalized effect: $N=R /\left(\left(R T_{A}+R T_{B}\right) / 2\right)$ with $R T_{X}$ representing mean $R T$ for condition $\mathrm{x})^{1}$. For all group data, participant scores outside the range of the mean $\pm 2.5 \mathrm{SD}$ of that group were removed from analysis.

\section{Statistical analyses}

\section{Depression, rumination and mindfulness skills scores}

ANCOVAs were run to test whether groups differed on depressive symptoms, rumination and mindfulness skills on the post-measurement. Group (MBCT versus TAU) was the between-subject factor of interest and either depression, rumination or mindfulness on the post-measurement was the dependent variable. In addition, either depression, rumination or mindfulness on the pre-measurement was entered as a covariate in the analysis to correct for possible differences between groups on these measures. In order to check whether depression diagnosis at the pre-measurement interfered with the effect of treatment on clinical outcome, depression diagnosis was entered as a fixed factor in the model. Of note, depression diagnosis did not interact with the effect of treatment as probed by either Hamilton or rumination scores $(p$-values of depression diagnosis $\times$ group interaction $>.1)$.

\footnotetext{
${ }^{1}$ Normalized network data were calculated by dividing the absolute network effect by the mean RT of the two conditions used to calculate the network effect. See Wang et al. for similar analyses of RT network scores (Wang et al., 2005).
} 


\section{Attention network scores}

ANCOVAs were run to test whether groups differed on ANT network scores (expressed in normalized RT and ES) at the post-measurement. One of the ANT networks was the dependent variable and group (MBCT versus TAU) was the between-subject factor of interest. In addition, Hamilton and attention network score of the pre-measurement were entered as covariates in the analysis to correct for possible differences between groups on these variables.

\section{Overall attentional processing}

Logistic regression analyses on overall data (all conditions combined) were run to test whether groups differently changed over time on more general, integrated attentional performance (see also Van den Hurk et al. (2010)), indexed by the performance on either target detection or response accuracy. In the first logistic regression analysis the aim was to probe the ability to detect the targets and so the dependent variable was response (yes versus no (= miss)) and the predictors were time and group. Of note, because the ANT has not been designed as a signal detection task, we expected to find very low numbers of misses in both groups (floor effects). However, we decided to run the analysis to check whether groups did not differ with respect to detection rates. In the second logistic regression analysis the aim was to probe the accuracy in responding and so the dependent variable was response (correct versus incorrect) and the predictors were time (pre versus post), group (MBCT versus TAU) and RTcen. RTcen is a variable of which the scores were obtained by a transformation of the variable RT. Each RT score was recalculated into a RTcen score by subtracting the overall mean RT score from this score. As such a new RTcen distribution was obtained with a mean RTcen score of zero. Because of this transformation, the outcomes of the logistic regression analyses show effects for the overall mean RT (instead of an RT of zero) and as such meaningful interpretations of the outcomes of the regression analysis were allowed for. The variable RTcen was included in the model as a predictor because RTcen is likely to explain much of the variance - RTcen is related to (predicts scores on) the dependent variable as reflected by the fact that with increasing reaction time the chance to respond accurately increases improving the power of the analysis.

\section{Results}

\section{Depression, rumination and mindfulness skills scores}

ANCOVA with HRSD post-scores showed a significant difference between groups, $F(1,65)=10.01, p<$ $.01, \eta^{2}=.13$. MBCT participants showed lower depression scores than controls on the postmeasurement when corrected for differences in HRSD pre-scores (see Table 1). In line with the relief of depressive symptoms, ANCOVA with rumination post-scores showed significantly lower rumination scores for the MBCT group after treatment, $F(1,63)=11.25, p<.01, \eta^{2}=.15$. ANCOVA with trainer as between-subject factor, either HRSD or rumination post-score as dependent variable and either HRSD or rumination pre-score as covariate, revealed no differences between trainers in clinical outcomes, $F(2$, $29)=.32, p=.73$ for HRSD scores and $F(2,28)=.15, p=.86$ for rumination scores. Finally, the MBCT patients scored higher on the observe, $F(1,65)=18.61, p<.001, \eta^{2}=.22$; act-with-awareness, $F(1,64)$ $=24.76, p<.001, \eta^{2}=.28$; and accept-without-judgment, $F(1,65)=22.10, p<.001, \eta^{2}=.25$, scales after treatment (see Table 1). On the describe scale MBCT patients did not score significantly higher after treatment, $\mathrm{F}(1,66)=1.72, p=.20$. 
Table 1: Estimated marginal means (SE) of the MBCT and control group at the post-measurement, F-values, $p$ values and effect sizes $\left(\eta^{2}\right)$ for HRSD, rumination and mindfulness skills are shown.

\begin{tabular}{lccccc}
\hline & Control & MBCT & F-value & $\mathbf{p}$ & $\mathbf{\eta}^{2}$ \\
\hline HRSD & $11.59(.95)$ & $7.12(1.03)$ & 10.01 & $<.01$ & .13 \\
Rumination & $28.39(1.14)$ & $22.72(1.24)$ & 11.25 & $<.01$ & .15 \\
Observe & $16.60(.91)$ & $22.46(1.00)$ & 18.61 & $<.001$ & .22 \\
Describe & $17.35(.56)$ & $18.42(.61)$ & 1.72 & .20 & .03 \\
Act-with-awareness & $14.90(.79)$ & $20.63(.84)$ & 24.76 & $<.001$ & .28 \\
Accept-without-judgment & $18.10(.69)$ & $22.95(.77)$ & 22.10 & $<.001$ & .25 \\
\hline
\end{tabular}

\section{Attention Networks analyses}

\section{Normalized RT scores}

None of the ANCOVAs with normalized RT data of the attentional networks on the post-measurement showed a significant difference between groups: for alerting, $F(1,65)=2.88, p=.10$, for orienting, $F(1$, 67 ) $=.26, p=.62$ and for executive attention, $F(1,65)=.65, p=.42$ (see Figure 2).

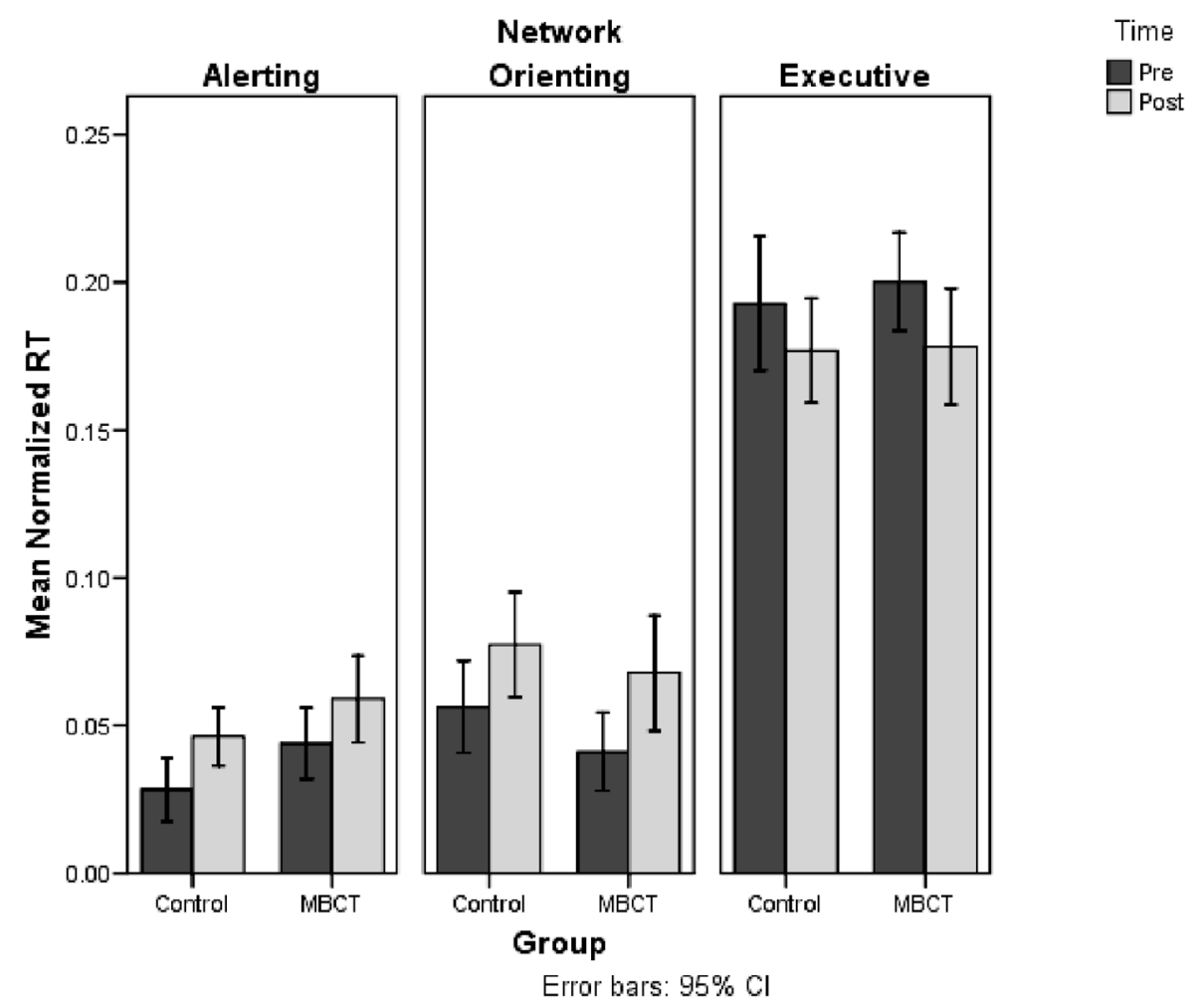

Figure 2: Normalized RT data of both groups, MBCT (+TAU) versus control (TAU), for the alerting, orienting and executive attention network of the pre- and post-measurement. Error bars indicate $95 \%$ confidence intervals (Cl). For all networks, ANCOVA showed no difference between groups in attention network scores on the postmeasurement when corrected for differences in attention network and Hamilton scores on the pre-measurement.

\section{Error scores}

None of the ANCOVAs with ES data of the attentional networks on the post-measurement showed a significant difference between groups: for alerting, $F(1,66)=1.30, p=.26$, for orienting, $F(1,65)=1.01$, $p=.32$ and for executive attention, $F(1,63)=.53, p=.47$ (see Figure 3 ). 


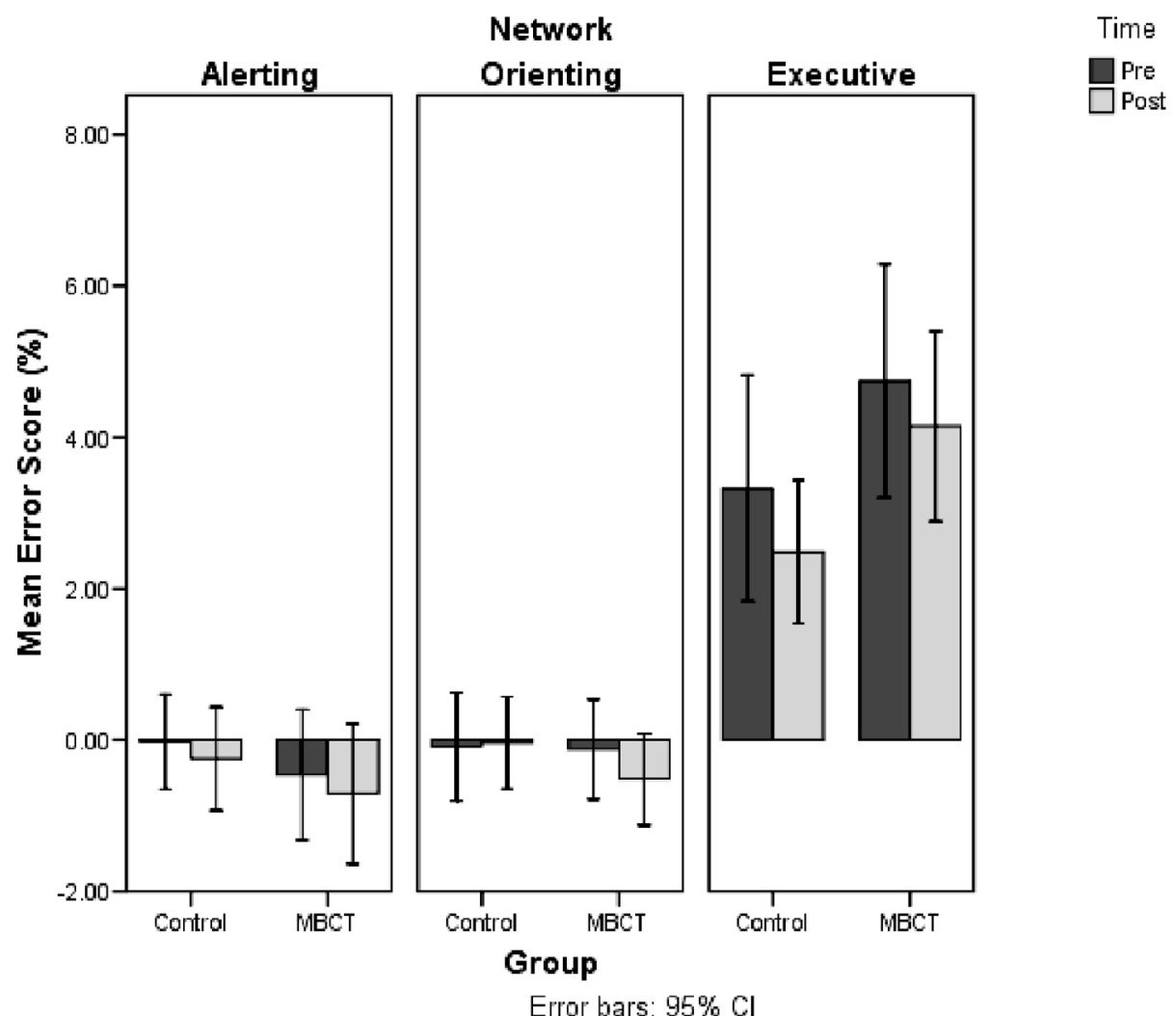

Figure 3: Error score data (\%) of both groups, MBCT (+TAU) versus control (TAU), for the alerting, orienting and executive attention network of the pre- and post-measurement. Error bars indicate 95\% confidence intervals (Cl). For all networks, ANCOVA showed no difference between groups in attention network scores on the postmeasurement when corrected for differences in attention network and Hamilton scores on the pre-measurement.

\section{Overall attentional performance}

\section{Probability of detections and misses}

The first logistic regression analysis was run to test whether overall attentional performance (expressed in terms of the chance of a response/miss) of the MBCT group differently changed over time. A significant Group (MBCT versus TAU) $\times$ Time (pre versus post) interaction would show this to be the case. In order to optimize the regression analysis for testing the significance of the Group $\times$ Time interaction, the other terms Time and Group were included. Neither Time, $p=.64$, nor Group, $p=.40$, nor the Group $\times$ Time interaction, $p=.48$, was a significant predictor. Thus, these results - the terms Group, Time and Group $\times$ Time not being significant predictors - showed that both groups did not differ from each other and did not (differently) change over time with respect to the number of misses, respectively.

\section{Probability of correct and incorrect responses}

The second logistic regression analysis was run to test whether overall attentional performance (expressed in terms of accuracy of response) of the MBCT group differently changed over time as compared to the control group. A significant Group (MBCT versus TAU) $\times$ Time (pre versus post) interaction would show this to be the case. In order to optimize the regression analysis for testing the significance of the Group $\times$ Time interaction, the other terms RTcen, Time, RTcen $\times$ Time, Group, Group $\times$ RTcen and Group $\times$ RTcen $\times$ Time were included. As expected, RTcen was a significant predictor, $\beta=$ 
$.011, p<.001, \operatorname{Exp}(B)=1.011$. No significant Group $\times$ Time interaction was found, $p=.30$, so the MBCT group did not show a different change in overall attentional processing over time. In summary, when either the chance of a miss or the accuracy of the response was considered, the MBCT group did not show a different change over time as compared to the TAU group.

\section{Discussion}

The aim of this study was to investigate whether attentional improvements are seen after MBCT in a sample of recurrently depressed patients and whether these improvements in attention mediate the efficacy of the MBCT program. The results show reductions in depressive symptoms and ruminative thinking and an increase in mindfulness skills after MBCT. These results are in line with previous studies that also showed lower depression and rumination scores and increased mindfulness skills after a shortterm mindfulness intervention (Anderson, et al., 2007; Giommi, et al., 2006; Kenny \& Williams, 2007; Kingston, et al., 2007; Michalak, Hölz, \& Teismann, 2011) and point to the efficacy of MBCT for recurrently depressed patients. Contrary to the clinical improvements, no differential changes in either specific, basal components of attention (alerting, orienting and executive attention) or in more general, integrated attentional processing are seen.

The finding of no improvements in specific components of attentional processing in our study is in line with the results obtained in other studies involving healthy participants that also did not show improvements in specific, basal components of attentional functioning after a short-term mindfulness training (Anderson, et al., 2007; Chambers, et al., 2008). To our knowledge, only in the study by Jha et al. (2007) a short-term mindfulness training (MBSR) has been associated with a change in a specific component of attentional processing (Jha et al., 2007), however limited to the orienting of attention. Long-term mindfulness practices, on the other hand, have consistently been associated with improvements in specific, basal components of attentional processing (Chan \& Woollacott, 2007; Moore \& Malinowski, 2009; Valentine \& Sweet, 1999; Van den Hurk, et al., 2010). In these studies, participants had significantly more meditation experience than the amount that can be acquired during either the eight weeks of MBCT, MBSR, or short-term mindfulness retreats. The larger amount of meditation experience in these studies might explain the improvements in basal components of attentional functioning, suggesting that such improvements might only be expected after extended periods of training, in both healthy and clinical populations. Further research is needed to investigate whether longterm mindfulness training can improve attentional processing in a clinical sample of recurrently depressed patients.

Both short-term and long-term mindfulness trainings have been associated with improvements at a more general, integrated level of attentional functioning (Anderson, et al., 2007; Chambers, et al., 2008; Slagter, et al., 2007; Van den Hurk, et al., 2010). Chambers et al. (2008) and Anderson et al. (2007) found changes in the overall performance on attention tasks - as reflected by improved information processing speed - after short-term mindfulness trainings, whereas Slagter et al. (2007) and van den Hurk et al. (2010) found similar improvements after long-term trainings. The outcome of the object detection task - during which participants had to indicate whether or not a common object was present in either a consistent or inconsistent scene - used to measure non-directed attention in the study by Anderson et al. led them to suggest that mindfulness may be more closely associated with changes in the 'quality of awareness of present moment experience' than with changes in specific, basal components of attentional functioning (Anderson, et al., 2007).

In the present study, participation in MBCT was not associated with a differential improvement in more general, integrated attentional functioning as measured with the ANT. The lack of finding a differential 
improvement in overall attentional processing is in contrast with our hypothesis and the results of the studies on short-term mindfulness trainings by Chambers et al. (2008) and Anderson et al. (2007). We speculate that the ANT might not be the appropriate task to probe improvements in overall attentional processing similar to those found by Chambers et al. (2008) and Anderson et al. (2007). In the study by Chambers and colleagues differences in sustained attention were shown by improved information processing speed in the internal switching task (IST) due to the intensive practice of mindfulness meditation. For the IST participants were instructed to maintain a silent mental count of either neutral or affective words belonging to two different categories and had to press the spacebar as soon as they had updated their mental count and were ready for the next word. In the study by Anderson and colleagues improvements in mindfulness after MBSR were correlated with improvements in object detection, as probed with the object detection task described above. Hence, these two attention tasks seem to be very different from the ANT in the way attentional processing is probed. The fact that the studied sample consisted of recurrently depressed patients might be an alternative explanation, as only healthy participants were tested in the studies by Chambers et al. (2008) and Anderson et al. (2007). Nonetheless, it seems likely that there is more room for improvement in attentional processing in a clinical sample of recurrently depressed patients that has previously been shown to have impairments in attentional processing than in a sample of healthy individuals (Koster, et al., 2010).

Contrary to our predictions, neither in basal components of attention nor in more general attentional processing were improvements observed after MBCT. It seems unlikely that the ANT was not the appropriate task to detect such improvements in attention as previous research, in which the ANT was also used, has related such improvements to the practice of mindfulness meditation by comparing the performance of only twenty mindfulness meditators to the performance of twenty matched controls (Van den Hurk, et al., 2010). Furthermore, it seems unlikely that low quality of delivery of MBCT might play a role in the null findings on attentional processing, since highly experienced MBCT trainers guided the groups. Moreover, the clinical results are compelling in showing the ability of the trainers to transfer the essentials of the MBCT teachings to the participants, as both depressive symptoms and rumination decreased after MBCT. Finally, also the lack of sufficient home practice seems not the reason for the null findings on attentional processing, since on average participants indicated to be engaged in formal as well as informal meditation for around 30 days during the MBCT program, i.e., every other day. Of note, the amount of home practice was not correlated to any of the attention network scores on the postmeasurement (all $p$-values $>.05$, not reported in results section).

Altogether, the results of our study suggest that improvements in attentional processing might not have a significant role in mediating the efficacy of MBCT for recurrently depressed patients. At this point, it might be meaningful to consider the operational definition of mindfulness as proposed by Bishop et al. (Bishop, et al., 2004). In their seminal paper, the psychological construct of mindfulness was conceptualized to consist of two components: the first component involves the self-regulation of attention so that it is maintained on immediate experience, thereby allowing for increased recognition of mental events in the present moment. The second component involves adopting a particular orientation toward one's experiences in the present moment, an orientation that is characterized by intentional curiosity, openness, and acceptance. Such an orientation seems tightly linked to the well-known idea of equanimity, i.e., reduced emotional reactivity, which is assumed to be associated with the practice of mindfulness meditation (Bodhi, 1993). As the first component seems closely related to the measurements of attentional functioning in our study, our data suggest that it might be especially the second component of mindfulness that has a mediating role in the efficacy of MBCT.

The suggestion that it might be the second component of mindfulness that is responsible for the positive clinical changes seems supported by the results of the studies by Kohls and colleagues and Kuyken and 
colleagues (Kohls, Sauer, \& Walach, 2009; Kuyken, et al., 2010). The findings by Kohls et al. suggested heuristic value in a two-factorial mindfulness construct, consisting of a 'presence' and an 'acceptance' factor and showed that the negative association between mindfulness and depression was completely due to the acceptance factor. Likewise, the study by Kuyken and colleagues showed that the effects of MBCT are mediated by enhancement of mindfulness and self-compassion, which are concepts that are strongly related to an attitude that is characterized by accepting and not judging oneself for having (negative) thoughts, emotions and inner feelings. In line with these findings, MBCT participants in our study also showed increases in mindfulness skills including, among others, accept-without-judgment. Moreover, the ability to accept-without-judgment was found to mediate the relationship between participation in MBCT and reduction of depressive symptoms in the total sample of recurrently depressed patients that was studied (Van Aalderen, et al.,2011) ${ }^{2}$. In the study by Verhoeven and others (Verhoeven et al., In submission) improved performance of recurrently depressed patients on the emotional Stroop task after MBCT was found. The emotional Stroop task measures interference from emotional stimuli and this might be related to emotional reactivity. Thus, the results from the study be Verhoeven and colleagues might provide (indirect) support for the idea that changes in emotional reactivity (second component of mindfulness), rather than changes in the self-regulation of attention (first component), have a mediating role in the efficacy of MBCT for patients with recurrent depression. Interestingly, these results are very much in line with the suggestion that was made in a recent review on cognitive mechanisms and depression (Koster, et al., 2010). In their paper, Koster and colleagues propose the impaired disengagement of attention to be central to the psychopathological mechanisms involved in depression, linking attention, rumination and clinical depression. According to their framework depression seems characterized by valence-specific impaired attentional control and that brooding - the more pathogenic aspect of rumination - has been correlated particularly with valence-specific inhibitory deficits. Thus, the cultivation of the second component of mindfulness - promoting an attitude of acceptance, openness and curiosity towards thoughts and emotions and fostering reduced emotional reactivity - appears a good candidate therapeutic factor able to specifically target this psychopathological mechanism. Further research is needed to investigate this hypothesis in more detail. In addition, it would be interesting to study the self-regulation of attention in the presence of emotional stimuli in recurrently depressed MBCT patients.

Noteworthy, a relatively large number of participants had previous experience with meditation: 17 out of 37 participants in the control group and 16 out of 34 participants in the MBCT group. No group difference was present regarding the number of participants with previous meditation experience $(p=.93)$, ruling out the possibility that differences in previous meditation experience account for the observed group differences on the clinical outcome variables. Still, it might be possible that previous meditation experience does interfere with the effect of treatment. In other words, the effect of treatment might be largest for the group of participants without any previous meditation experience. By means of additional analyses (ANCOVA with previous meditation experience as additional factor; not reported in results section) we checked whether previous meditation experience did interact with the effect of treatment on clinical outcome. It turned out that previous meditation experience did not interact with the effect of MBCT on either depressive symptoms or rumination ( $p$-values $>.1)$. The latter finding might be due to the fact that the majority of participants with previous experience had only relatively little experience; 27 out of the 33 participants with previous experience had only less than 5 years of experience.

\footnotetext{
${ }^{2}$ For a further elaboration on these clinical and mindfulness skills data, please see the study by van Aalderen et al. (2011).
} 


\section{Concluding remarks}

Altogether, the results of the present study suggest that improvements in attentional processing, observed after long-term mindfulness trainings, are not found after a short-term training (MBCT) in a clinical sample of recurrently depressed patients. These findings suggest that the clinical improvements, seen after MBCT, might not be primarily mediated by improvements in the self-regulation of attention (i.e., the first component of mindfulness according to Bishop et al. (2004)). Together with recent, converging research findings they suggest that it might be mostly the second component of mindfulness according to Bishop et al. (2004) - referring to the cultivation of an attitude that is characterized by openness and acceptance and related to reduced emotional reactivity - that mediates the efficacy of MBCT for recurrently depressed patients. It would be interesting for future research to inquire this hypothesis more deeply and to explore whether this hypothesis can be generalized to mindfulnessbased interventions for other clinical samples.

\section{Acknowledgements}

The authors gratefully acknowledge the support of the BrainGain Smart Mix Programme of the Netherlands Ministry of Economic Affairs and the Netherlands Ministry of Education, Culture and Science and the support of Fonds Psychische Gezondheid (Grant Number: 2005 6028). In addition, we thank Cobie Wijsman and Tom Wingens for their help during data acquisition.

This work was conducted at Department of Psychiatry, Radboud University Nijmegen Medical Centre and at Faculty of Science, Radboud University Nijmegen, the Netherlands.

\section{References}

Anderson, N. D., Lau, M. A., Segal, Z. V., \& Bishop, S. R. (2007). Mindfulness-based stress reduction and attentional control. Clinical Psychology and Psychotherapy, 14, 449-463. http://dx.doi.org/10.1002/cpp.544

APA. (2000). Diagnostic and Statistical Manual of Mental Disorders. Fourth Edition. Text Revision. DSMIV-TR. Washington DC: American Psychiatric Association.

Baer, R. A., Smith, G. T., \& Allen, K. B. (2004). Assessment of mindfulness by self-report: the Kentucky inventory of mindfulness skills. Assessment, 11(3), 191-206. http://dx.doi.org/10.1177/1073191104268029

Baer, R. A., Smith, G. T., Hopkins, J., Krietemeyer, J., \& Toney, L. (2006). Using self-report assessment methods to explore facets of mindfulness. Assessment, 13(1), 27-45. http://dx.doi.org/10.1177/1073191105283504

Baum, C., Kuyken, W., Bohus, M., Heidenreich, T., Michalak, J., \& Steil, R. (2010). The Psychometric Properties of the Kentucky Inventory of Mindfulness Skills in Clinical Populations. Assessment 17, 220-229. http://dx.doi.org/10.1177/1073191109356525

Beck, A. T., Rush, A. J., Shaw, B. F., \& Emery, G. (1979). Cognitive therapy of depression. New York: Guilford Press.

Bishop, S. R., Lau, M., Shapiro, S., Carlson, L., Anderson, N. D., Carmody, J.,... Devins, G. (2004). Mindfulness: A proposed operational definition. Clinical Psychology: Science and Practice, 11, 230241. http://dx.doi.org/10.1093/clipsy.bph077

Bodhi, B. (1993). Abhidhammattha Sangaha. A comprehensive manual of abhidhamma. Seattle, USA: BPS Pariyatti Editions. 
Chambers, R., Lo, B. C. Y., \& Allen, N. B. (2008). The impact of intensive mindfulness training on attentional control, cognitive style, and affect. Cognitive Therapy and Research, 32, 303-322. http://dx.doi.org/10.1007/s10608-007-9119-0

Chan, D., \& Woollacott, M. (2007). Effects of level of meditation experience on attentional focus: is the efficiency of executive or orientation networks improved? The Journal of Alternative and Complementary Medicine, 13(6), 651-657. http://dx.doi.org/10.1089/acm.2007.7022

Conway, M., Csank, P. A. R., Holm, S. L., \& Blake, C. K. (2000). On assessing individual differences in rumination on sadness. Journal of Personality Assessment, 75(3), 404-425.

http://dx.doi.org/10.1207/S15327752JPA7503 04

Dekeyser, M., Raes, F., Leijssen, M., Leysen, S., \& Dewulf, D. (2008). Mindfulness skills and interpersonal behavior. Personality and Individual Differences, 44, 1235-1245. http://dx.doi.org/10.1016/i.paid.2007.11.018

Fan, J., McCandliss, B. D., Sommer, T., Raz, A., \& Posner, M. I. (2002). Testing the efficiency and independence of attentional networks. Journal of Cognitive Neuroscience, 14, 340-347. http://dx.doi.org/10.1162/089892902317361886

First, M. B., Spitzer, R. L., Gibbon, M., \& Williams, J. B. W. (1995). Structured clinical interview for DSMIV axis I disorders-patient edition (SCID-I/P, Version 2.0). Washington, DC: American Psychiatric Press.

Giommi, F., Barendregt, H. P., Oliemeulen, L., van Hoof, J., Tinge, J., Coenen, A., et al. (2006). Mindfulness-based trainings and emotional disorders. In M. G. T. Kwee, K. J. Gergen \& F. Koshikawa (Eds.), Horizons In Buddhist Psychology: Practice, Research and Theory (pp. 213-224). Chagrin Falls, Ohio, USA: Taos Institute Publications.

Groenestijn, M. A. C., Akkerhuis, G. W., Kupka, R. W., Schneider, N., \& Nolen, W. A. (1999). Structured clinical interview for DSM-IV axis I disorders. Lisse, the Netherlands: Swets.

Hamilton, M. (1960). A rating scale for depression. Journal of Neurosurgery and Psychiatry, 23(1), 5662. http://dx.doi.org/10.1136/jnnp.23.1.56

Heeren, A., van Broeck, N., \& Philippot, P. (2009). The effects of mindfulness on executive processes and autobiographical memory specificity. Behaviour Research and Therapy, 47, 403-409. http://dx.doi.org/10.1016/j.brat.2009.01.017

Hermans, P. J., van Ree, F., Betgem, P., \& Westenbrink, A. (1981). Betrouwbaarheid en validiteit van het Hamilton Interview [Reliability and validity of the Hamilton Interview] Gedrag: tijdschrift voor psychologie, 9, 219-233.

Jha, A. P., Krompinger, J., \& Baime, M. J. (2007). Mindfulness training modifies subsystems of attention. Cognitive, Affective \& Behavioral Neuroscience, 7(2), 109-119. http://dx.doi.org/10.3758/CABN.7.2.109

Joormann, J., \& Gotlib, I. H. (2007). Selective attention to emotional faces following recovery from depression. Journal of Abnormal Psychology, 116, 80-85. http://dx.doi.org/10.1037/0021$\underline{843 X .116 .1 .80}$

Kabat-Zinn, J. (1990). Full catastrophe living: The program of the Stress Reduction Clinic at the University of Massachusetts Medical Center. New York: Dell Publishing.

Kenny, M. A., \& Williams, J. M. G. (2007). Treatment-resistant depressed patients show a good response to Mindfulness-based Cognitive Therapy. Behaviour Research and Therapy, 45, 617-625. http://dx.doi.org/10.1016/j.brat.2006.04.008

Kingston, T., Dooley, B., Bates, A., Lawlor, E., \& Malone, K. (2007). Mindfulness-based cognitive therapy for residual depressive symptoms. Psychology and Psychotherapy: Theory, Research and Practice, 80, 193-203. 
Kohls, N., Sauer, S., \& Walach, H. (2009). Facets of mindfulness - results of an online study investigating the Freiburg mindfulness inventory. Personality and Individual Differences, 46, 224-230. http://dx.doi.org/10.1016/j.paid.2008.10.009

Koster, E. H. W., De Lyssnyder, E., Derakshan, N., \& De Raedt, R. (2010). Understanding depressive rumination from a cognitive science perspective: The impaired disengagement hypothesis. Clinical Psychology Review, In Press.

Kuyken, W., Byford, S., Taylor, R. S., Watkins, E., Holden, E., White, K.,... Teasdale, J. D. (2008). Mindfulness-based cognitive therapy to prevent relapse in recurrent depression. Journal of Consulting and Clinical Psychology, 76, 966-978. http://dx.doi.org/10.1037/a0013786

Kuyken, W., Watkins, E., Holden, E., White, K., Taylor, R. S., Byford, S., .. Dalgleish, T. (2010). How does mindfulness-based cognitive therapy work? Behaviour Research and Therapy, 48, 1105-1112. http://dx.doi.org/10.1016/j.brat.2010.08.003

Ma, S. H., \& Teasdale, J. D. (2004). Mindfulness-based cognitive therapy for depression: replication and exploration of differential relapse prevention effects. Journal of Consulting and Clinical Psychology, 72, 31-40. http://dx.doi.org/10.1037/0022-006X.72.1.31

Merriam, E. P., Thase, M. E., Haas, G. L., Keshavan, M. S., \& Sweeney, J. A. (1999). Prefrontal cortical dysfunction in depression determined by Wisconsin card sorting performance. American Journal of Psychiatry, 156(780-782).

Michalak, J., Heidenreich, T., Meibert, P., \& Schulte, D. (2008). Mindfulness predicts relapse/recurrence in major depressive disorder after mindfulness-based cognitive therapy. The Journal of Nervous and Mental Disease, 196(8), 630-633. http://dx.doi.org/10.1097/NMD.0b013e31817d0546

Michalak, J., Hölz, A., \& Teismann, T. (2011). Rumination as a predictor of relapse in Mindfulness-Based Cognitive Therapy for depression. Psychology and Psychotherapy: Theory, Research and Practice, 84, 230-236.

Moore, A., \& Malinowski, P. (2009). Meditation, mindfulness and cognitive flexibility. Consciousness and Cognition, 18, 176-186. http://dx.doi.org/10.1016/j.concog.2008.12.008

Raes, F., Hermans, D., \& Eelen, P. (2003). De Nederlandstalige versie van de Ruminative Response Scale (RRS-NL) en de Rumination on Sadness Scale (RRS-NL) [ The Dutch version of the Ruminative Response Scale (RRS-NL) and the Rumination on Sadness Scale (RRS-NL]. Gedragstherapie, 36, 97-104.

Segal, Z., Bieling, P., Young, T., Macqueen, G., Cooke, R., Martin, L.,... Levitan, R.D. (2010). Antidepressant Monotherapy vs Sequential Pharmacotherapy and Mindfulness-Based Cognitive Therapy, or Placebo, for Relapse Prophylaxis in Recurrent Depression. Archives of General Psychiatry, 67, 1256-1264. http://dx.doi.org/10.1001/archgenpsychiatry.2010.168

Segal, Z. V., Williams, J. M. G., \& Teasdale, J. D. (2002). Mindfulness-based cognitive therapy for depression: A new approach to preventing relapse. New York: Guilford Press.

Sheehan, D. V., Lecrubier, Y., Sheehan, K. H., Amorim, P., Janavs, J., Weiller, E.,... Dunbar G. C. (1998). The Mini-International Neuropsychiatric Interview (MINI): the development and validation of a structured diagnostic psychiatric interview for DSM-IV and ICD-10. Journal of Clinical Psychiatry, 59, 22-33.

Slagter, H. A., Lutz, A., Greischar, L. L., Francis, A. D., Nieuwenhuis, S., Davis, J. M., \& Davidson R. J. (2007). Mental training affects distribution of limited brain resources. PLoS Biology 5(6). http://dx.doi.org/10.1371/journal.pbio.0050138

Teasdale, J.D., Segal, Z., Williams, J.M.G. (1995) How does cognitive therapy prevent depressive relapse and why should attentional control (mindfulness) training help? Behaviour Research and Therapy, 33(1), 25-39. http://dx.doi.org/10.1016/0005-7967(94)E0011-7 
Teasdale, J. D., Segal, Z. V., Williams, J. M. G., Ridgeway, V. A., Soulsby, J. M., \& Lau, M. A. (2000). Prevention of relapse/recurrence in major depression by mindfulness-based cognitive therapy. Journal of Consulting and Clinical Psychology, 68, 615-623. http://dx.doi.org/10.1037/0022$\underline{006 X .68 .4 .615}$

Valentine, E. R., \& Sweet, P. L. G. (1999). Meditation and attention: A comparison of the effects of concentrative and mindfulness meditation on sustained attention. Mental Health, Religion \& Culture, 2(1), 59-70. http://dx.doi.org/10.1080/13674679908406332

Van Aalderen, J.R., Donders, A.R.T., Giommi, F., Spinhoven, P., Barendregt, H.P., and Speckens, A.E.M. (2011). The efficacy of mindfulness-based cognitive therapy in recurrent depressed patients with and without a current depressive episode: a randomized controlled trial. Psychological Medicine. http://dx.doi.org/10.1017/S0033291711002054

Van den Hurk, P. A. M., Giommi, F., Gielen, S. C., Speckens, A. E. M., \& Barendregt, H. P. (2010). Greater efficiency in attentional processing related to mindfulness meditation. Quarterly Journal of Experimental Psychology, 63(6), 1168-1180. http://dx.doi.org/10.1080/17470210903249365

Van Vliet, I. M., Leroy, H., \& Van Megen, H. (2000). MINI Plus: MINI Internationaal Neuropsychiatrisch Interview: Dutch version 5.0.0. Leiden: LUMC.

Verhoeven, J. E., Vrijsen, J. N., van Oostrom, I., Speckens, A. E. M., Janzing, J. G. E., \& Rincka, M. (In submission). Attentional Control Effects of Mindfulness-Based Cognitive Therapy in Formerly Depressed Patients.

Wang, K., Fan, J., Dong, Y., Wang, C. Q., Lee, T. M., \& Posner, M. I. (2005). Selective impairment of attentional networks of orienting and executive control in schizophrenia. Schizophrenia Research, 78(2-3), 235-241. http://dx.doi.org/10.1016/j.schres.2005.01.019 